\title{
AUTOMORPHISMS OF ORDER 4 OF THE SIMPLY CONNECTED COMPACT LIE GROUP $E_{6}$
}

\author{
By \\ Ichiro Yokota and Osamu Shukuzawa
}

Using the theory of Kac-Moody Lie algebras, for compact simple Lie algebra $g$, automorphisms $\rho$ of finite order of $g$ can be classified and the type of Lie subalgebras $g^{\rho}$ of fixed points are determined [1]. Now for the simply connected compact Lie group $E_{6}$, we realize automorphisms $\rho$ of order 4 and determine the subgroups $\left(E_{6}\right)^{\rho}$ of fixed points. Among compact exceptional Lie groups, only $E_{6}$ has outer automorphisms, so we consider the case of $E_{6}$. As results, the group $E_{6}$ has eight inner automorphisms named as $\gamma_{1}, \gamma_{2}, \cdots, \gamma_{5}, \sigma_{1}$, $\sigma_{2}, \sigma_{3}$ and three outer automorphisms named as $\tau \gamma_{2}{ }^{\prime}, \tau \gamma_{3}, \tau \sigma_{3}$, and the subgroups $\left(E_{6}\right)^{\rho}$ of fixed points are given as follows.

\begin{tabular}{|c|c|c|}
\hline$\rho$ & $\left(e_{6}\right)^{\rho}$ & $\left(E_{6}\right)^{\rho}$ \\
\hline$\gamma_{1}$ & $T^{1} \oplus A_{1} \oplus A_{4}$ & $(S p(1) \times S(U(1) \times U(5))) / Z_{2}$ \\
\hline$\sigma_{2}$ & $T^{1} \oplus A_{1} \oplus A_{1} \oplus A_{3}$ & $(S p(1) \times S(U(2) \times U(4))) / \boldsymbol{Z}_{2}$ \\
\hline$\gamma_{2}$ & $T^{1} \oplus A_{1} \oplus A_{2} \oplus A_{2}$ & $(S p(1) \times S(U(3) \times U(3))) / Z_{2}$ \\
\hline$\gamma_{3}$ & $T^{1} \oplus A_{5}$ & $(U(1) \times S U(6)) / Z_{2}$ \\
\hline$\gamma_{4}$ & $T^{2} \oplus A_{4}$ & $(U(1) \times S(U(1) \times U(5))) / Z_{2}$ \\
\hline$\gamma_{5}$ & $T^{2} \oplus A_{1} \oplus A_{3}$ & $(U(1) \times S(U(2) \times U(4))) / Z_{2}$ \\
\hline$\sigma_{1}$ & $T^{1} \oplus D_{5}$ & $(U(1) \times \operatorname{Spin}(10)) / Z_{4}$ \\
\hline$\sigma_{3}$ & $T^{2} \oplus D_{4}$ & $(U(1) \times(\operatorname{Spin}(2) \times \operatorname{Spin}(8))) /\left(\boldsymbol{Z}_{2} \times \boldsymbol{Z}_{4}\right)$ \\
\hline$\tau \gamma_{2}^{\prime}$ & $A_{1} \oplus D_{3}$ & $(S p(1) \times S O(6)) / Z_{2}$ \\
\hline$\tau \gamma_{3}$ & $T^{1} \oplus C_{3}$ & $(U(1) \times S p(3)) / Z_{2}$ \\
\hline$\tau \sigma_{3}$ & $A_{1} \oplus B_{3}$ & $(S U(2) \times \operatorname{Spin}(7)) / Z_{2}$. \\
\hline
\end{tabular}

\section{Preliminaries}

Let $\boldsymbol{F}=\boldsymbol{H} \oplus \boldsymbol{H} \boldsymbol{e}(\boldsymbol{H}$ is the field of quaternions with the basis $\{1, \boldsymbol{i}, \boldsymbol{j}, \boldsymbol{k}\})$ be the Cayley algebra with the multiplication $(m+a e)(n+b e)=(m n-\bar{b} a)+(a \bar{n}+b m) e$, the conjugation $\overline{m+a e}=\bar{m}-a e$, the inner product $(x, y)=(\bar{x} y+\bar{y} x) / 2$ and the length $|x|=\sqrt{(x, x)}$, and $\varsigma^{c}$ be its complexification. Let $\Im=\left\{X \in M(3, \S) \mid X^{*}=X\right\}$

Received September 10, 1990. Revised March 7, 1991. 
be the exceptional Jordan algebra with the multiplication $X \circ Y=(X Y+Y X) / 2$ and $\Im^{C}$ be its complexification. $\Im^{\prime}$ and $\Im^{C}$ have the inner product $(X, Y)=\operatorname{tr}(X \circ Y)$, the Freudenthal multiplication $X \times Y=(2 X \circ Y-\operatorname{tr}(X) Y-\operatorname{tr}(Y) X+(\operatorname{tr}(X) \operatorname{tr}(Y)$ $-(X, Y)) E) / 2$ and the determinant $\operatorname{det} X=(X \times X, X) / 3 . \quad(\Im(3, \boldsymbol{H})=\{M \in M(3, \boldsymbol{H}) \mid$ $\left.M^{*}=M\right\}$ and $\Im(3, \boldsymbol{H})^{C}$ are also defined). The complex conjugations of $\varsigma^{c}, \Im^{C}$ are denoted by $\tau$. In $\mathcal{S}^{C}$, the positive definite inner product $\langle X, Y\rangle$ is defined by $(\tau X, Y)$. Now

$$
\begin{aligned}
E_{6} & =\left\{\alpha \in \operatorname{Iso}_{C}\left(\Im^{C}\right) \mid \operatorname{det} \alpha X=\operatorname{det} X,\langle\alpha X, \alpha Y\rangle=\langle X, Y\rangle\right\} \\
& =\left\{\alpha \in \operatorname{Iso}_{C}\left(\Im^{C}\right) \mid \alpha X \times \alpha Y=\tau \alpha \tau(X \times Y),\langle\alpha X, \alpha Y\rangle=\langle X, Y\rangle\right\}
\end{aligned}
$$

is the simply connected compact Lie group of type $E_{6}$ [2]. Throughout this paper, we use such notations and theorems in [4] as $E, E_{i}, F_{i}\left(x_{i}\right), i=1,2,3$ of $\Im$, $\Im^{C}$ and Lie subgroups $F_{4}=\left\{\alpha \in E_{6} \mid \tau \alpha=\alpha \tau\right\}=\left\{\alpha \in E_{6} \mid \alpha E=E\right\}, \operatorname{Spin}(9)=$ $\left\{\alpha \in F_{4} \mid \alpha E_{1}=E_{1}\right\}, \operatorname{Spin}(10)=\left\{\alpha \in E_{6} \mid \alpha E_{1}=E_{1}\right\}$ of $E_{6}$ etc. .

\section{Inner automorphisms $\gamma_{1}, \gamma_{2}, \cdots, \gamma_{5}$ of order 4 of $E_{6}$}

The field $\boldsymbol{H}$ is embedded in $M(2, \boldsymbol{C})$ by $k: \boldsymbol{H}=\boldsymbol{C} \oplus \boldsymbol{C} \boldsymbol{j} \rightarrow M(2, \boldsymbol{C})$ (where $\boldsymbol{C}=$ $\{x+y \boldsymbol{i} \mid x, y \in \boldsymbol{R}\})$ by $k(a+b \boldsymbol{j})=\left(\begin{array}{rr}a & b \\ -\bar{b} & \bar{a}\end{array}\right), a, b \in \boldsymbol{C} . \quad$ This $k$ is naturally extended to $\boldsymbol{R}$-linear mappings $k: M(3, \boldsymbol{H}) \rightarrow M(6, \boldsymbol{C}), k: \boldsymbol{H}^{3} \rightarrow M(2,6, \boldsymbol{C})$. Moreover these $k$ are extended to $C$ - $\boldsymbol{C}$-linear isomorphisms $k: M(3, \boldsymbol{H})^{C} \rightarrow M(6, \boldsymbol{C}), \quad k:\left(\boldsymbol{H}^{3}\right)^{C} \rightarrow$ $M(2,6, C)$

$$
\begin{aligned}
& k\left(M_{1}+i M_{2}\right)=k\left(M_{1}\right)+i k\left(M_{2}\right), \quad M_{i} \in M(3, \boldsymbol{H}), \\
& k\left(\boldsymbol{a}_{1}+i \boldsymbol{a}_{2}\right)=k\left(\boldsymbol{a}_{1}\right)+\boldsymbol{i} k\left(\boldsymbol{a}_{2}\right) \quad \boldsymbol{a}_{i} \in \boldsymbol{H}^{3} .
\end{aligned}
$$

Finally we define the $\boldsymbol{C}$-vector space $\mathfrak{S}(6, \boldsymbol{C})$ by $\left\{\left.S \in M(6, \boldsymbol{C})\right|^{t} S=-S\right\}$ and the $C$-C-linear isomorphism $k_{J}: \Im(3, \boldsymbol{H})^{C} \rightarrow \Im(6, \boldsymbol{C})$ by

$$
k_{J}\left(M_{1}+i M_{2}\right)=k\left(M_{1}\right) J+i k\left(M_{2}\right) J, \quad M_{i} \in \Im(3, \boldsymbol{H})
$$

where $J=\operatorname{diag}(J, J, J), J=\left(\begin{array}{rr}0 & 1 \\ -1 & 0\end{array}\right)$.

In $\Im(3, \boldsymbol{H})^{C} \oplus\left(\boldsymbol{H}^{3}\right)^{C}$, we define the Freudenthal multiplication [2] as

$$
(M+\boldsymbol{a}) \times(N+\boldsymbol{b})=\left(M \times N-\frac{1}{2}\left(\boldsymbol{a}^{*} \boldsymbol{b}+\boldsymbol{b}^{*} \boldsymbol{a}\right)\right)-\frac{1}{2}(\boldsymbol{a} N+\boldsymbol{b} M) .
$$

Then $\Im^{C}$ is isomorphic to $\Im(3, \boldsymbol{H})^{C} \oplus\left(\boldsymbol{H}^{3}\right)^{C}$ by the correspondence 


$$
\left(\begin{array}{ccc}
\xi_{1} & x_{3} & \bar{x}_{2} \\
\bar{x}_{3} & \xi_{2} & x_{1} \\
x_{2} & \bar{x}_{1} & \xi_{3}
\end{array}\right) \longleftrightarrow\left(\begin{array}{ccc}
\xi_{1} & m_{3} & \bar{m}_{2} \\
\bar{m}_{3} & \xi_{2} & m_{1} \\
m_{2} & \bar{m}_{1} & \xi_{3}
\end{array}\right)+\left(a_{1}, a_{2}, a_{3}\right)
$$

(where $x_{i}=m_{i}+a_{i} e, m_{i}, a_{i} \in \boldsymbol{H}^{C}$ ) as Freudenthal algebra [4]. Hereafter we identify $\Im^{C}$ and $\Im(3, \boldsymbol{H})^{C} \oplus\left(\boldsymbol{H}^{3}\right)^{C}$. We define an involutive $C$-linear mapping $\gamma: \mathfrak{S}^{C} \rightarrow \mathfrak{\Im}^{C}$ by

$$
\gamma(M+\boldsymbol{a})=M-\boldsymbol{a}, \quad M+\boldsymbol{a} \in \mathfrak{\Im}(3, \boldsymbol{H})^{C} \oplus\left(\boldsymbol{H}^{3}\right)^{C}=\mathfrak{\Im}^{C} .
$$

Then $\gamma \in E_{6}$ and $\gamma^{2}=1$.

Proposition 2.1. $\left(E_{6}\right)^{r} \cong(S p(1) \times S U(6)) / \boldsymbol{Z}_{2}, \boldsymbol{Z}_{2}=\{(1, E),(-1,-E)\}$.

Proof. Let $S p(1)=\{p \in \boldsymbol{H} \mid \bar{p} p=1\}$ and $S U(6)=\left\{A \in M(6, \boldsymbol{C}) \mid A^{*} A=E \operatorname{det} A\right.$ $=1\}$. Now the mapping $\phi: S p(1) \times S U(6) \rightarrow\left(E_{6}\right)^{\gamma}$,

$$
\phi(p, A)(M+\boldsymbol{a})=k_{J}^{-1}\left(A k_{J}(M)^{t} A\right)+p k^{-1}\left(k(\boldsymbol{a}) A^{*}\right), \quad M+\boldsymbol{a} \in \mathfrak{J}^{C}
$$

induces the required isomorphism. The details of proof are in [2] or [4].

REMARK. $\quad \phi: S p(1) \times S U(6) \rightarrow\left(E_{6}\right)^{\gamma}$ satisfies $\gamma=\psi(-1, E)$ and $\tau \psi(p, A) \tau=$ $\phi(p,-J \bar{A} J)^{\prime}$

Using $\phi: S p(1) \times S U(6) \rightarrow\left(E_{6}\right)^{r}$ of Proposition 2.1 , we define

$$
\begin{array}{ll}
\gamma_{1}=\psi\left(1, \boldsymbol{i} I_{1}\right), & I_{1}=\operatorname{diag}(-1,1,1,1,1,1), \\
\gamma_{2}=\psi\left(1, \boldsymbol{i} I_{3}\right), & I_{3}=\operatorname{diag}(-1,-1,-1,1,1,1), \\
\gamma_{3}=\psi(\boldsymbol{i}, E), & \\
\gamma_{4}=\psi\left(\varepsilon, \boldsymbol{\varepsilon} \Gamma_{1}\right), & \boldsymbol{\varepsilon}=(1+\boldsymbol{i}) / \sqrt{2}, \Gamma_{1}=\operatorname{diag}(\boldsymbol{i}, 1,1,1,1,1), \\
\gamma_{5}=\psi\left(\boldsymbol{i}, I_{2}\right), & I_{2}=\operatorname{diag}(-1,-1,1,1,1,1) .
\end{array}
$$

Then $\gamma_{i} \in E_{6}$ and the order of $\gamma_{i}$ is 4 , for $i=1,2, \cdots, 5$.

THEOREM 2.2. (1) $\left(E_{6}\right)^{\gamma_{1}} \cong(S p(1) \times S(U(1) \times U(5))) / \boldsymbol{Z}_{2}$,

(2) $\left(E_{6}\right)^{r_{2}} \cong(S p(1) \times S(U(3) \times U(3))) / Z_{2}$,

(3) $\left(E_{6}\right)^{r_{3}} \cong(U(1) \times S U(6)) / \boldsymbol{Z}_{2}$,

(4) $\left(E_{6}\right)^{\gamma_{5}} \cong(U(1) \times S(U(2) \times U(4))) / Z_{2}$

where $Z_{2}=\{(1, E),(-1,-E)\}$ in any case.

PRoOF. (1) Since $\gamma_{1}^{2}=\gamma$, we have $\left(E_{6}\right)^{\gamma_{1}} \subset\left(E_{6}\right)^{\gamma}$. Hence, for $\alpha \in\left(E_{6}\right)^{\gamma_{1}}$ there exist $p \in S p(1), A \in S U(6)$ such that $\alpha=\psi(p, A)$ (Proposition 2.1). From the 
condition $\gamma_{1} \alpha=\alpha \gamma_{1}$, we have $\phi\left(p, i I_{1} A\right)=\psi\left(p, i A I_{1}\right)$, that is, $I_{1} A=A I_{1}$, therefore $A \in S(U(1) \times U(5))$. Thus we have the required isomorphism.

(2), (3), (4) are proved to be similar to (1).

THEOREM 2.3. $\left(E_{6}\right)^{\gamma_{4}} \cong(U(1) \times S(U(1) \times U(5))) / Z_{2}, Z_{2}=\{(1, E),(-1,-E)\}$.

Proof. Since the operation of $\gamma_{4}$ on $\mathfrak{\Im}^{C}=\mathfrak{\$}(3, \boldsymbol{H})^{C} \oplus\left(\boldsymbol{H}^{3}\right)^{C}$ is given by

$$
\begin{aligned}
& \left.\gamma_{4}\left(\begin{array}{ccc}
\xi_{1} & m_{3} & \bar{m}_{2} \\
\bar{m}_{3} & \xi_{2} & m_{1} \\
m_{2} & \bar{m}_{1} & \xi_{3}
\end{array}\right)+\left(a_{1}, a_{2}, a_{3}\right)\right) \\
& =\left(\begin{array}{ccc}
-\xi_{1} & i \varepsilon \varepsilon m_{3} & i \varepsilon \varepsilon \bar{m}_{2} \\
i \varepsilon \bar{m}_{3} \bar{\varepsilon} & i \xi_{2} & i m_{1} \\
i \varepsilon m_{2} \bar{\varepsilon} & i \bar{m}_{1} & i \xi_{3}
\end{array}\right)+\left(-i \varepsilon a_{1} \bar{\varepsilon},-i \varepsilon \varepsilon a_{2},-i \varepsilon \varepsilon a_{3}\right)
\end{aligned}
$$

(where $\varepsilon=(1+i) / \sqrt{2}$ ), the eigen $C$-vector spaces $\left(\Im^{C}\right)_{\nu}, \nu=1,-1, i,-i$ with respect to $\gamma_{4}$ are

$$
\begin{aligned}
& \left(\Im^{C}\right)_{1}=\left\{M+\boldsymbol{a} \in \Im(3, \boldsymbol{H})^{C} \oplus\left(\boldsymbol{H}^{3}\right)^{C} \mid \gamma_{4}(M+\boldsymbol{a})=M+\boldsymbol{a}\right\} \\
& =\left\{\left(a_{1}(\boldsymbol{i}-i),(\boldsymbol{i}+i) a_{2},(\boldsymbol{i}+i) a_{3}\right) \mid a_{1} \in \boldsymbol{C} \boldsymbol{j}, a_{2}, a_{3} \in \boldsymbol{H}\right\}, \\
& \left(\Im^{C}\right)_{-1}=\left\{M+\boldsymbol{a} \in \mathfrak{\$}(3, \boldsymbol{H})^{C} \oplus\left(\boldsymbol{H}^{3}\right)^{C} \mid \gamma_{4}(M+\boldsymbol{a})=-M-\boldsymbol{a}\right\} \\
& =\left\{\left(\begin{array}{ccc}
\boldsymbol{\xi}_{1} & (\boldsymbol{i}+i) m_{3} & \overline{m_{2}(\boldsymbol{i}-i)} \\
\overline{(\boldsymbol{i}+i) m_{3}} & 0 & 0 \\
m_{2}(\boldsymbol{i}-i) & 0 & 0
\end{array}\right)+\left.\left(a_{1}(\boldsymbol{i}+i), 0,0\right)\right|_{a_{1} \in \boldsymbol{C} \boldsymbol{j}, m_{2}, m_{3} \in \boldsymbol{H}} ^{\boldsymbol{\xi}_{1} \in C,},\right. \\
& \left(\Im^{C}\right)_{i}=\left\{M+\boldsymbol{a} \in \Im(3, \boldsymbol{H})^{C} \oplus\left(\boldsymbol{H}^{3}\right)^{C} \mid \gamma_{4}(M+\boldsymbol{a})=i(M+\boldsymbol{a})\right\} \\
& =\left\{\left.\left(\begin{array}{ccc}
0 & (\boldsymbol{i}-i) m_{3} & \overline{m_{2}(\boldsymbol{i}+i)} \\
\overline{(\boldsymbol{i}-i) m_{3}} & \boldsymbol{\xi}_{2} & m_{1} \\
m_{2}(\boldsymbol{i}+i) & \bar{m}_{1} & \boldsymbol{\xi}_{3}
\end{array}\right)\right|_{m_{1} \in \boldsymbol{H}^{c}, m_{2}, m_{3} \in \boldsymbol{H}}\right\}, \\
& \left(\mathfrak{S}^{C}\right)_{-i}=\left\{M+\boldsymbol{a} \in \mathfrak{\Im}(3, \boldsymbol{H})^{C} \oplus\left(\boldsymbol{H}^{3}\right)^{C} \mid \gamma_{4}(M+\boldsymbol{a})=-i(M+\boldsymbol{a})\right\} \\
& \left.=\left\{\left(a_{1},(\boldsymbol{i}-i) a_{2},(\boldsymbol{i}-i) a_{3}\right)\right) \mid a_{1} \in \boldsymbol{C}^{C}, a_{2}, a_{3} \in \boldsymbol{H}\right\}
\end{aligned}
$$

where $\boldsymbol{C} \boldsymbol{j}=\{s \boldsymbol{j}+t \boldsymbol{k} \mid s, t \in \boldsymbol{R}\}$. These spaces are invariant under the group $\left(E_{6}\right)^{\gamma_{4}}$. We shall show that $\left(\boldsymbol{H}^{3}\right)^{C}$ is invariant under $\left(E_{6}\right)^{r_{4}}$. From the forms of $\left(\mathfrak{S}^{C}\right)_{\nu}$, it is sufficient to show that $\alpha \boldsymbol{a} \in\left(\boldsymbol{H}^{3}\right)^{C}$ for $\alpha \in\left(E_{6}\right)^{\gamma_{4}}$ and $\boldsymbol{a}=(a(\boldsymbol{i}+i), 0,0)=$ $F_{1}((a(\boldsymbol{i}+i)) e)(a \in \boldsymbol{C} \boldsymbol{j})$. Now, in fact, 


$$
\begin{aligned}
& \alpha F_{1}((a(\boldsymbol{i}+i)) e)=4 \alpha\left(\left(F_{3}((\boldsymbol{i}-i) \bar{a}) \times F_{1}(1)\right) \times F_{3}(e)\right) \\
& \quad=4\left(\alpha F_{3}((\boldsymbol{i}-i) \bar{a}) \times \alpha F_{1}(1)\right) \times \tau \alpha \tau F_{3}(e) \\
& \quad \in 4\left(\Im(3, \boldsymbol{H})^{C} \times \Im(3, \boldsymbol{H})^{C}\right) \times\left(\boldsymbol{H}^{3}\right)^{C} \subset \Im(3, \boldsymbol{H})^{C} \times\left(\boldsymbol{H}^{3}\right)^{C} \subset\left(\boldsymbol{H}^{3}\right)^{C} .
\end{aligned}
$$

Thus we see that $\left(\boldsymbol{H}^{3}\right)^{C}$ is invariant under $\left(E_{6}\right)^{\gamma_{4}}$, hence $\Im(3, \boldsymbol{H})^{C}=\left(\left(\boldsymbol{H}^{3}\right)^{C}\right)^{\perp}=$ $\left\{X \in \Im^{C} \mid\langle X, Y\rangle=0\right.$ for all $\left.Y \in\left(\boldsymbol{H}^{3}\right)^{C}\right\}$ is also invariant under $\left(E_{6}\right)^{r_{4}}$. Consequently, $\alpha \in\left(E_{6}\right)^{\gamma_{4}}$ commutes with $\gamma$, that is, $\left(E_{6}\right)^{\gamma_{4}} \subset\left(E_{6}\right)^{\gamma}$. Hence, for $\alpha \in\left(E_{6}\right)^{\gamma_{4}}$, there exist $p \in S p(1), A \in S U(6)$ such that $\alpha=\phi(p, A)$ (Proposition 2.1). From the condition $\gamma_{4} \alpha=\alpha \gamma_{4}$, we have $\phi\left(\varepsilon p, \varepsilon \Gamma_{1} A\right)=\phi\left(p \varepsilon, A \varepsilon \Gamma_{1}\right)$, that is, $\varepsilon p=p \varepsilon, \Gamma_{1} A$ $=A \Gamma_{1}$ (or $\varepsilon p=-p \varepsilon, \Gamma_{1} A=-A \Gamma_{1}$ (which is impossible)), therefore $p \in U(1)$, $A \in S(U(1) \times U(5))$. Thus we have the required isomorphism.

\section{Inner automorphisms $\sigma_{1}, \sigma_{2}, \sigma_{3}$ of order 4 of $E_{6}$}

Let $U(1)=\{\theta \in C \mid(\tau \theta) \theta=1\}$ (where $C=\boldsymbol{R}^{C}$ ) and we define an embedding $\phi$ : $U(1) \rightarrow E_{6}$ by

and put $\sigma=\phi(-1) \in E_{6}$.

$$
\phi(\theta)\left(\begin{array}{ccc}
\xi_{1} & x_{3} & \bar{x}_{2} \\
\bar{x}_{3} & \xi_{2} & x_{1} \\
x_{2} & \bar{x}_{1} & \xi_{3}
\end{array}\right)=\left(\begin{array}{ccc}
\theta^{4} \xi_{1} & \theta x_{3} & \theta \bar{x}_{2} \\
\theta \bar{x}_{3} & \theta^{-2} \xi_{2} & \theta^{-2} x_{1} \\
\theta x_{2} & \theta^{-2} \bar{x}_{1} & \theta^{-2} \xi_{3}
\end{array}\right)
$$

The group $\operatorname{Spin}(10)$ is defined by $\left(E_{6}\right)_{E_{1}}=\left\{\alpha \in E_{6} \mid \alpha E_{1}=E_{1}\right\}$ which is the covering group of $S O(10)=S O\left(V^{10}\right)$ where $V^{10}=\left\{X \in \mathcal{S}^{C} \mid 2 E_{1} \times X=-\tau X\right\}=$ $\left\{\xi E_{2}-\tau \xi E_{3}+F_{1}(x) \mid \xi \in C, x \in \mathbb{F}\right\}$. Note that $\operatorname{Spin}(10)$ leaves invariant $\left\{X \in \mathfrak{\Im}^{C} \mid\right.$ $\left.E_{1} \times X=0\right\}=\left\{F_{2}(x)+F_{3}(y) \mid x, y \in \mathbb{E}^{C}\right\}$.

Proposition 3.1. $\left(E_{6}\right)^{\sigma} \cong(U(1) \times \operatorname{Spin}(10)) / \boldsymbol{Z}_{4}, \boldsymbol{Z}_{4}=\{(1,1),(-1, \sigma),(i, \phi(-i))$, $(-i, \phi(i))\}$.

Proof. The mapping $\varphi: U(1) \times \operatorname{Spin}(10) \rightarrow\left(E_{6}\right)^{\sigma}$,

$$
\varphi(\theta, \beta)=\phi(\theta) \beta
$$

induces the required isomorphism. The details of proof are in [2] or [4].

Using $\phi: U(1) \rightarrow E_{6}$ or $\phi: S p(1) \times S U(6) \rightarrow E_{6}$ of Proposition 2.1, we define

$$
\begin{aligned}
& \sigma_{1}=\phi(i)=\phi\left(-1, \Gamma_{4}\right), \quad \Gamma_{4}=\operatorname{diag}(1,1, \boldsymbol{i}, \boldsymbol{i}, \boldsymbol{i}, \boldsymbol{i}), \\
& \sigma_{2}=\gamma \sigma_{1}=\psi\left(1, \Gamma_{4}\right) .
\end{aligned}
$$

Then $\sigma_{i} \in E_{6}$ and $\sigma_{i}{ }^{2}=\sigma, \sigma_{i}{ }^{4}=1$ for $i=1,2$.

THEOREM 3.2. $\left(E_{6}\right)^{\sigma_{1}} \cong(U(1) \times \operatorname{Spin}(10)) / \boldsymbol{Z}_{4}, \boldsymbol{Z}_{4}=\langle(i, \phi(-i))\rangle$. 
ProOF is clear from Proposition 3.1 , because $\sigma_{1}=\phi(i)$ commutes with any elements of $U(1)$ and $\operatorname{Spin}(10)$.

THEOREM 3.3. $\left(E_{6}\right)^{\sigma_{2}} \cong(S p(1) \times S(U(2) \times U(4))) / \boldsymbol{Z}_{2}, \boldsymbol{Z}_{2}=\{(1, E),(-1,-E)\}$.

Proof. Since $\sigma_{2}{ }^{2}=\sigma$, we have $\left(E_{6}\right)^{\sigma_{2}} \subset\left(E_{6}\right)^{\sigma}$. Hence, for $\alpha \in\left(E_{6}\right)^{\sigma_{2}}$, there exist $\theta \in U(1), \beta \in \operatorname{Spin}(10)$ such that $\alpha=\phi(\theta) \beta$ (Proposition 3.1). In particular, $\alpha$ commutes with $\sigma_{1}=\phi(i)$. Therefore, from the condition $\sigma_{2} \alpha=\alpha \sigma_{2}$, that is, $\gamma \sigma_{1} \alpha=\alpha \gamma \sigma_{1}$, we have $\gamma \alpha=\alpha \gamma$, namely $\alpha \in\left(E_{6}\right)^{\gamma}$. Hence there exist $p \in S p(1)$, $A \in S U(6)$ such that $\alpha=\psi(p, A)$ (Proposition 2.1). Moreover from the condition $\sigma_{1} \alpha=\alpha \sigma_{1}$, we have $\phi\left(p, \Gamma_{4} A\right)=\psi\left(p, A \Gamma_{4}\right)$, that is, $\Gamma_{4} A=A \Gamma_{4}$, therefore $A \in$ $S(U(2) \times U(4))$. Thus we have the required isomorphism.

REMARK. The group $\left(E_{6}\right)^{\sigma_{2}}$ has also the following expression

$$
\left(E_{6}\right)^{\sigma_{2}} \cong(U(1) \times S p(1) \times(S U(2) \times S U(4))) /\left(Z_{2} \times Z_{4}\right)
$$

where $\boldsymbol{Z}_{2}=\left\langle\left(1,-1,-I_{2}\right)\right\rangle, \boldsymbol{Z}_{4}=\left\langle\left(-i, 1,-\Gamma_{4}\right)\right\rangle$. In fact, for $\alpha=\psi(p, A) \in\left(E_{6}\right)^{\sigma_{2}}$, $p \in S p(1), A=(P, Q)=\left(\begin{array}{ll}P & 0 \\ 0 & Q\end{array}\right) \in S(U(2) \times U(4))$, the condition that $\alpha$ belongs to the group $\left(\left(E_{6}\right)^{\sigma_{2}}\right)_{E_{1}} \subset \operatorname{Spin}(10)$, that is, $\psi(p,(P, Q)) E_{1}=E_{1}$, is $p \in S p(1), P \in S U(2)$, $Q \in S U(4)$. From this we have easily the required isomorphism.

The field $\boldsymbol{C}$ of complex numbers is embedded in $\mathbb{E}$ as $\boldsymbol{C}=\{x+y e \mid x, y \in \boldsymbol{R}\}$ and put $C^{\perp}=\{t \in \mathbb{E} \mid(t, C)=0\}$. Let $\operatorname{Spin}(2)=\{a \in C \mid \bar{a} a=1\}(\cong U(1))$ and we define an embedding $D: \operatorname{Spin}(2) \rightarrow E_{6}$ by

$$
D_{a}\left(\begin{array}{ccc}
\xi_{1} & x_{3} & \bar{x}_{2} \\
\bar{x}_{3} & \xi_{2} & x_{1} \\
x_{2} & \bar{x}_{1} & \xi_{3}
\end{array}\right)=\left(\begin{array}{ccc}
\xi_{1} & x_{3} \bar{a} & \bar{x}_{2} a \\
a \bar{x}_{3} & \xi_{2} & a x_{1} a \\
\bar{a} x_{2} & \overline{a x_{1} a} & \xi_{3}
\end{array}\right) .
$$

Put $\sigma_{3}=D_{-e}$. Then $\sigma_{3} \in E_{6}$ and $\sigma_{3}{ }^{2}=\sigma, \sigma_{3}{ }^{4}=1$.

The group $\operatorname{Spin}(8)$ is defined by

$$
\begin{aligned}
\left(E_{6}\right)_{E_{1}, F_{1}(s)} & =\left\{\alpha \in E_{6} \mid \alpha E_{1}=E_{1}, \alpha F_{1}(s)=F_{1}(s) \text { for all } s \in C^{C}\right\} \\
& =\left\{\alpha \in \operatorname{Spin}(10) \mid \alpha F_{1}(1)=F_{1}(1), \alpha F_{1}(e)=F_{1}(e)\right\}
\end{aligned}
$$

which is the covering group of $S O(8)=S O\left(V^{8}\right)$ where $V^{8}=\left(V^{10}\right)_{\sigma_{3}}=\left\{\xi E_{2}-\tau \xi E_{3}\right.$ $\left.+F_{1}(t) \mid \xi \in C, t \in C^{\perp}\right\}$.

LEMMA 3.3. $D_{a}(a \in \operatorname{Spin}(2))$ and $\beta \in \operatorname{Spin}(8)$ commute with each other. 
PROOF.

$$
\begin{aligned}
\beta D_{a} F_{1}(z) & =\beta F_{1}(a z a)=\beta F_{1}\left(a^{2} s+t\right) \quad\left(z=s+t \in C^{C} \oplus\left(\boldsymbol{C}^{\perp}\right)^{C}=\mathfrak{c}^{C}\right) \\
& =F_{1}\left(a^{2} s\right)+\beta F_{1}(t)=F_{1}\left(a^{2} s\right)+\left(\xi_{2} E_{2}+\xi_{3} E_{3}+F_{1}\left(t^{\prime}\right)\right) \quad\left(\xi_{i} \in C, t^{\prime} \in\left(\boldsymbol{C}_{-}^{\perp}\right)^{C}\right) \\
& =D_{a}\left(F_{1}(s)+\xi_{2} E_{2}+\xi_{3} E_{3}+F_{1}\left(t^{\prime}\right)\right)=D_{a}\left(F_{1}(s)+\beta F_{1}(t)\right) \\
& =D_{a} \beta F_{1}(s+t)=D_{a} \beta F_{1}(z) . \\
\beta D_{a} F_{2}(z) & =\beta F_{2}(\bar{a} z)=4 \beta\left(\left(F_{1}(1) \times F_{2}(z)\right) \times F_{1}(a)\right)=4\left(\beta F_{1}(1) \times \beta F_{2}(z)\right) \times \tau \beta \tau F_{1}(a) \\
& =4\left(F_{1}(1) \times\left(F_{2}(x)+F_{3}(y)\right) \times F_{1}(a) \quad\left(\text { for some } x, y \in \Im^{C}\right)\right. \\
& =F_{2}(\bar{a} x)+F_{3}(y \tilde{a})=D_{a}\left(F_{2}(x)+F_{3}(y)\right)=D_{a} \beta F_{2}(z) .
\end{aligned}
$$

Similarly $\beta D_{a} F_{3}(z)=D_{a} \beta F_{3}(z)$. Clearly $D_{a} \beta=\beta D_{a}$ on $E_{1}$.

$$
D_{a} \beta E_{2}=D_{a}\left(\xi_{2} E_{2}+\xi_{3} E_{3}+F_{1}(t)\right)=\xi_{2} E_{2}+\xi_{3} E_{3}+F_{1}(t)=\beta E_{2}=\beta D_{a} E_{2}
$$

(for some $\xi_{i} \in C, t \in\left(\boldsymbol{C}^{\perp}\right)^{C}$ ). Similarly $D_{a} \beta E_{3}=\beta D_{a} E_{3}$. Thus we have $D_{a} \beta$ $=\beta D_{a}$.

LemMA 3.4. Let $\beta \in(\operatorname{Spin}(10))^{\sigma_{3}}$. Then we can put $\beta F_{1}(1)=F_{1}(s), \beta F_{1}(e)=$ $F_{1}(e s), s \in C,|s|=1$.

Proof. Since the group $(\operatorname{Spin}(10))^{\sigma_{3}}$ acts on $\left\{F_{1}(s) \mid s \in C\right\}=\left\{X \in \mathfrak{S}^{C} \mid \sigma_{3} X=\right.$ $\left.-X, 2 E_{1} \times X=-\tau X\right\}$, we can put

$$
\beta F_{1}(1)=F_{1}(s), \quad \beta F_{1}(e)=F_{1}\left(s^{\prime}\right), \quad s, s^{\prime} \in C,|s|=\left|s^{\prime}\right|=1 .
$$

Operate $\tau \beta \tau$ on the relation $F_{1}(1) \times F_{1}(e)=-(1, e) E_{1}=0$, then $0=\tau \beta \tau\left(F_{1}(1) \times F_{1}(e)\right.$ ). $=\beta F_{1}(1) \times \beta F_{1}(e)=F_{1}(s) \times F_{1}\left(s^{\prime}\right)=-\left(s, s^{\prime}\right) E_{1}$, hence $\left(s, s^{\prime}\right)=0$. Together with $|s|$ $=\left|s^{\prime}\right|=1$, we have $s^{\prime}=e s$ or $s^{\prime}=-e s$. The latter case is impossible. In fact, choose $s \in C$ such that $a^{2}=\bar{s}$ and put $\delta=D_{a} \beta$, then $\delta F_{1}(1)=F_{1}(1), \delta F_{1}(e)=-F_{1}(e)$. Then

$$
\begin{aligned}
\delta F_{2}(e) & =\delta \sigma_{3} F_{2}(1)=\sigma_{3} \delta F_{2}(1)=\sigma_{3}\left(F_{2}(x)+F_{3}(y)\right) \quad\left(\text { for some } x, y \in \varsigma^{C}\right) \\
& =F_{2}(e x)+F_{3}(y e) \\
\delta F_{3}(1) & =2 \delta\left(F_{1}(1) \times F_{2}(1)\right)=2 \tau \delta \tau F_{1}(1) \times \tau \delta \tau F_{2}(1) \\
& =2 F_{1}(1) \times\left(F_{2}(\tau x)+F_{3}(\tau y)\right)=F_{3}(\tau \bar{x})+F_{2}(\tau \bar{y}) .
\end{aligned}
$$

Therefore we have

$$
\begin{aligned}
F_{1}(e) & =\delta F_{1}(-e)=2 \delta\left(F_{2}(e) \times F_{3}(1)\right)=2 \tau \delta \tau F_{2}(e) \times \tau \delta \tau F_{3}(1) \\
& =2\left(F_{2}(e(\tau x))+F_{3}((\tau y) e) \times\left(F_{3}(\bar{x})+F_{2}(\bar{y})\right)\right. \\
& =F_{1}(-x((\tau \bar{x}) e)-(e(\tau \bar{y})) y)+* E_{2}+* E_{3} .
\end{aligned}
$$


Compare the coefficients of $e$, then we have $1=-\left|x_{1}\right|^{2}-\left|x_{2}\right|^{2}-\left|y_{1}\right|^{2}-\left|y_{2}\right|^{2}$ (where $x=x_{1}+i x_{2}, y=y_{1}+i y_{2}, x_{i}, y_{i} \in \mathbb{E}$ ), which is a contradiction. Thus Lemma 3.4 is proved.

THEOREM 3.5. $\left(E_{6}\right)^{\sigma_{3}} \cong(U(1) \times \operatorname{Spin}(2) \times \operatorname{Spin}(8)) /\left(\boldsymbol{Z}_{2} \times \boldsymbol{Z}_{4}\right)$,

$$
\begin{aligned}
Z_{2} \times Z_{4}= & \langle(1,-1, \sigma)\rangle \times\left\langle\left(i, e, \phi(i) D_{e}\right)\right\rangle \\
= & \left\{(1,1,1),(-1,1, \sigma),\left(i, e, \phi(i) D_{e}\right),\left(-i, e, \phi(-i) D_{e}\right),\right. \\
& \left.(1,-1, \sigma),(-1,-1,1),\left(i,-e, \phi(-i) D_{e}\right),\left(-i,-e, \phi(i) D_{e}\right)\right\} .
\end{aligned}
$$

Proof. We define a mapping $\phi: U(1) \times \operatorname{Spin}(2) \times \operatorname{Spin}(8) \rightarrow\left(E_{6}\right)^{\sigma_{3}}$ by

$$
\phi(\theta, a, \delta)=\phi(\theta) D_{a} \delta .
$$

Obviously $\phi$ is well-defined. Since $\phi(\theta)(\theta \in U(1)), D_{a}(a \in \operatorname{Spin}(2))$ and $\delta \in \operatorname{Spin}(8)$ commute with one another (Lemma 3.3), $\phi$ is a homomorphism. We shall show that $\psi$ is onto. (Although it suffices to show $\operatorname{dim}\left(\left(e_{6}\right)^{\sigma_{3}}\right)=30$, we will give a direct proof). Since $\sigma_{3}{ }^{2}=\sigma$, we have $\left(E_{6}\right)^{\sigma_{3}} \subset\left(E_{6}\right)^{\sigma}$. Hence, for $\alpha \in\left(E_{6}\right)^{\sigma_{3}}$, there exist $\theta \in U(1), \beta \in S p i n(10)$ such that $\alpha=\phi(\theta) \beta$ (Proposition 3.1). From $\sigma_{3} \alpha=\alpha \sigma_{3}$, we have $\beta \in(\operatorname{Spin}(10))^{\sigma_{3}}$. Hence we can put $\beta F_{1}(1)=F_{1}(s), \beta F_{1}(e)=F_{1}(e s), s \in C$, $|s|=1$ (Lemma 3.4). Choose $a \in C$ such that $a^{2}=s$ and put $\delta=D_{a}^{-1} \beta$, then $\delta F_{1}(1)=F_{1}(1), \delta F_{1}(e)=F_{1}(e)$, that is, $\delta \in \operatorname{Spin}(8)$. Hence we have a presentation such that

$$
\alpha=\phi(\theta) D_{a} \delta, \quad \theta \in U(1), a \in \operatorname{Spin}(2), \delta \in \operatorname{Spin}(8) .
$$

Then $\psi$ is onto. $\operatorname{Ker} \psi=Z_{2} \times Z_{4}$ is easily obtained. Thus we have the required isomorphism.

\section{Outer automorphisms $\tau \gamma_{2}{ }^{\prime}, \tau \gamma_{3}$ of oder 4 of $E_{6}$}

Using $\phi: S p(1) \times S U(6) \rightarrow\left(E_{6}\right)^{\gamma}$ of Proposition 2.1, we define $\gamma_{2}{ }^{\prime}=\psi(1, J)$ and consider an automorphism $\tau \gamma_{2}{ }^{\prime}$ of $E_{6}: E_{6} \ni \alpha \rightarrow \tau \gamma_{2}{ }^{\prime} \alpha \gamma_{2}{ }^{\prime-1} \tau \in E_{6}$. Then $\left(\tau \gamma_{2}{ }^{\prime}\right)^{2}=\gamma$, $\left(\tau \gamma_{2}{ }^{\prime}\right)^{4}=1$.

THEOREM 4.1. $\left(E_{6}\right)^{\tau \gamma_{2}}{ }^{\prime} \cong(S p(1) \times S O(6)) / Z_{2}, Z_{2}=\{(1, E),(-1,-E)\}$.

Proof. Since $\left(\tau \gamma_{2}{ }^{\prime}\right)^{2}=\gamma$, we have $\left(E_{6}\right)^{\tau \gamma_{2}{ }^{\prime}} \subset\left(E_{6}\right)^{\gamma}$. Hence, for $\alpha \in\left(E_{6}\right)^{\tau \gamma^{\prime}}{ }^{\prime}$, there exist $p \in S p(1), A \in S U(6)$ such that $\alpha=\phi(p, A)$ (Proposition 2.1). Since $\tau \gamma_{2}{ }^{\prime} \alpha \gamma_{2}{ }^{\prime-1} \tau=\alpha$, we have $\tau \psi(p,-J A J) \tau=\psi(p, A)$, that is, $\psi(p, \bar{A})=\psi(p, A)(\operatorname{Re}-$ mark of Proposition 2.1) then $A=\bar{A}$, hence $A \in S O(6)$. Thus we have the required isomorphism. 
We use $\gamma_{3}=\psi(i, E)$ of Section 2 and consider an automorphism $\tau \gamma_{3}$ of $E_{6}: E_{6}$ $\ni \alpha \rightarrow \tau \gamma_{3} \alpha \gamma_{3}{ }^{-1} \tau \in E_{6}$. Then $\left(\tau \gamma_{3}\right)^{2}=\gamma,\left(\tau \gamma_{3}\right)^{4}=1$.

THEOREM 4.2. $\left(E_{6}\right)^{\tau \gamma_{3}} \cong(U(1) \times S p(3)) / \boldsymbol{Z}_{2}, \boldsymbol{Z}_{2}=\{(1, E),(-1,-E)\}$.

ProOF. Since $\left(\tau \gamma_{3}\right)^{2}=\gamma$, we have $\left(E_{6}\right)^{\tau \gamma_{3}} \subset\left(E_{6}\right)^{\gamma}$. Hence, for $\alpha \in\left(E_{6}\right)^{\tau \gamma_{3}}$, there exist $p \in S p(1), A \in S U(6)$ such that $\alpha=\phi(p, A)$ (Proposition 2.1). Since $\tau \gamma_{3} \alpha \gamma_{3}{ }^{-1} \tau$ $=\alpha$, we have $\psi(-i p i,-J \bar{A} J)=\phi(p, A)($ Remark of Proposition 2.1), then

$$
p=-i p i, A=-J \bar{A} J \text { or } p=i p i, A=J \bar{A} J \text {. }
$$

In the first case $p \in U(1)=\{p \in C \mid \bar{p} p=1\}, A \in S p(3)=\left\{A \in M(6, C) \mid J A=\bar{A} J, A^{*} A\right.$ $=E$, $(\operatorname{det} A=1)\}$ (where $\boldsymbol{C} \subset \boldsymbol{H}$ ). The latter case is impossible. In fact, consider $A I$ where $I=\operatorname{diag}(1,-1,1,-1,1,-1)$, then $A I \in S p(3)$, hence $\operatorname{det}(A I)$ $=1$. On the other hand, $\operatorname{det}(A I)=(\operatorname{det} A)(\operatorname{det} I)=1(-1)=-1$, which contradicts $\operatorname{det}(A I)=1$. Thus we have the required isomorphism.

\section{Outer automorphism $\tau \sigma_{3}$ of order 4 of $E_{6}$}

As in Section 3, we embed tho field $\boldsymbol{C}$ of complex numbers in $\mathbb{E}$ as $\boldsymbol{C}=$ $\{x+y e \mid x, y \in \boldsymbol{R}\}$. Let $S U(2)=\left\{A \in M(2, \boldsymbol{C}) \mid A^{*} A=E\right.$, det $\left.A=1\right\}$ and we define a mapping $\phi: S U(2) \rightarrow E_{6}$ by

$$
\phi(A) X=\left(\rho_{1}(A)\right) X\left(\rho_{1}(A)\right)^{*}, \quad X \in \Im^{C}
$$

where $\rho_{1}(A)=\Gamma^{-1} A^{\prime} \Gamma, \Gamma=\operatorname{diag}(i, 1, i), A^{\prime}=\left(\begin{array}{ll}1 & 0 \\ 0 & A\end{array}\right)$. Explicitly, for $A=\left(\begin{array}{rr}a & -\bar{b} \\ b & \bar{a}\end{array}\right)$ $\in S U(2), z \in \mathbb{S}^{C}$,

$$
\begin{aligned}
& \left\{\begin{array}{l}
\phi(A) E_{1}=E_{1} \\
\phi(A)\left(E_{2}+E_{3}\right)=\left(|a|^{2}-|b|^{2}\right)\left(E_{2}+E_{3}\right)-2 i F_{1}(a \bar{b}) \\
\phi(A)\left(E_{2}-E_{3}\right)=E_{2}-E_{3}
\end{array}\right. \\
& \left\{\begin{array}{l}
\phi(A) F_{1}(z)=-2 i(z, \overline{b a})\left(E_{2}+E_{3}\right)+F_{1}(a z a-\overline{b z b}) \\
\phi(A) F_{2}(z)=F_{2}(\bar{a} z)-i F_{3}(\bar{z} b) \\
\phi(A) F_{3}(z)=-i F_{2}(b \bar{z})+F_{3}(z \bar{a})
\end{array}\right.
\end{aligned}
$$

Note that $D_{a}=\phi\left(\begin{array}{ll}a & 0 \\ 0 & \bar{a}\end{array}\right)$ for $a \in C,|a|=1$, in particular, $\phi\left(\begin{array}{rr}-e & 0 \\ 0 & e\end{array}\right)=D_{-e}=\sigma_{3}$.

Lemma 5.1. $\phi$ is well-defined, that is, for $A \in S U(2)$ we have $\phi(A) \in E_{6}$.

Proof. Define $\rho \in E_{6}$ by $\rho X=\Gamma X \Gamma, X \in \Im^{C}$. We consider an embedding 
$S U(3) \subset F_{4} \subset E_{6}$, in which $S U(3) \subset F_{4}$ is given by $h: S U(3) \rightarrow F_{4}$,

$$
h(A)(X+M)=A X A^{*}+M A^{*}, \quad X+M \in \mathfrak{S}\left(3, C^{C}\right) \oplus M\left(3, C^{C}\right)=\mathfrak{S}^{C}
$$

(as for notations see [5]). The $\phi(A)$ is nothing but $\phi(A)=\rho^{-1} h\left(A^{\prime}\right) \rho \in E_{6}$.

We use $\sigma_{3}$ of Section 3 and consider an automorphism $\tau \sigma_{3}$ of $E_{6}: E_{6} \ni \alpha \rightarrow$ $\tau \sigma_{3} \alpha \sigma_{3}{ }^{-1} \tau \in E_{6}$. Then $\left(\tau \sigma_{3}\right)^{2}=\sigma,\left(\tau \sigma_{3}\right)^{4}=1$.

LeMMA 5.2. For $\alpha \in\left(E_{6}\right)^{\tau \sigma_{3}}$, we have $\alpha E_{1}=E_{1}$. In particular, $\left(E_{6}\right)^{\tau \sigma_{3}} \subset\left(E_{6}\right)_{E_{1}}$ $=\operatorname{Spin}(10)$.

Proof. Since $\left(\tau \sigma_{3}\right)^{2}=\sigma$, we have $\left(E_{6}\right)^{\tau \sigma_{3}} \subset\left(E_{6}\right)^{\sigma}$. Hence, for $\alpha \in\left(E_{6}\right)^{\tau \sigma_{3}}$, there exists $\xi \in C,(\tau \xi) \xi=1$ such that $\alpha E_{1}=\xi E_{1}$ (Proposition 3.1). From $\xi E_{1}=$ $\alpha E_{1}=\alpha \tau \sigma_{3} E_{1}=\tau \sigma_{3} \alpha E_{1}=\tau \sigma_{3}\left(\xi E_{1}\right)=\tau \xi E_{1}$, we have $\tau \xi=\xi$, that is, $\xi \in \boldsymbol{R}$, hence $\xi= \pm 1$. The case of $\xi=-1$ is impossible. (Although it follows from the connectedness of $\left(E_{6}\right)^{\tau \sigma_{3}}$, we will give an elementary proof). Suppose $\xi=-1$. Let $\alpha=\phi(\theta) \beta, \theta \in U(1), \beta \in \operatorname{Spin}(10)$. Then $-E_{1}=\alpha E_{1}=\phi(\theta) \beta E_{1}=\theta^{4} E_{1}$, hence $\theta^{4}=$ -1. Now, for $t \in C^{\perp}$, we can put $\beta F_{1}(t)=\eta E_{2}-\tau \eta E_{3}+F_{1}(x), \eta \in C, x \in \mathbb{C}$. Then $\phi(\phi) \beta F_{1}(t)= \pm i \eta E_{2} \mp i \tau \eta E_{3} \pm i F_{1}(x)$. Since $\tau \sigma_{3} \alpha=\alpha \tau \sigma_{3}$, we have $\eta \in i \boldsymbol{R}$, $x \in C$. This shows that for $V=\left\{F_{1}(t) \mid t \in C^{\perp}\right\}, \operatorname{dim} V=6, \operatorname{dim}(\alpha V) \leqq 3$, which contradicts the regularity of $\alpha$. Thus Lemma 5.2 is proved.

The group $\operatorname{Spin}(7)$ is defined by

$$
\begin{aligned}
\left(E_{6}\right)_{E_{1}, E, F_{1}(s)} & =\left\{\alpha \in E_{6} \mid \alpha E_{1}=E_{1}, \alpha E=E, \alpha F_{1}(s)=F_{1}(s) \text { for all } s \in C\right\} \\
& =\left\{\alpha \in F_{4} \mid \alpha E_{1}=E_{1}, \alpha F_{1}(s)=F_{1}(s) \text { for all } s \in C\right\} \\
& =\left\{\alpha \in \operatorname{Spin}(9) \mid \alpha F_{1}(1)=F_{1}(1), \alpha F_{1}(e)=F_{1}(e)\right\}
\end{aligned}
$$

which is the covering group of $S O(7)=S O\left(V^{7}\right)$ where $V^{7}=\left\{\xi\left(E_{2}-E_{3}\right)+F_{1}(t) \mid\right.$ $\left.\xi \in \boldsymbol{R}, t \in \boldsymbol{C}^{\perp}\right\}$.

LEMMA 5.3. $\phi(A) \in \phi(S U(2))$ and $\beta \in S p i n(7)$ commute with each other.

Proof. For $A=\left(\begin{array}{rr}a & -\bar{b} \\ b & \bar{a}\end{array}\right) \in S U(2), \beta \in \operatorname{Spin}(7)$, we shall show

$$
\beta \phi(A) X=\phi(A) \beta X, \quad X \in \Im^{C} .
$$

$$
\begin{aligned}
\beta \phi(A)\left(E_{2}-E_{3}\right) & =\beta\left(E_{2}-E_{3}\right)=\xi\left(E_{2}-E_{3}\right)+F_{1}(t) \quad\left(\text { for some } \xi \in C, t \in\left(C^{\perp}\right)^{C}\right) \\
& =\phi(A)\left(\xi\left(E_{2}-E_{3}\right)+F_{1}(t)\right)=\phi(A) \beta\left(E_{2}-E_{3}\right),
\end{aligned}
$$




$$
\begin{aligned}
\beta \phi(A)\left(E_{2}+E_{3}\right) & =\beta\left(\left(|a|^{2}-|b|^{2}\right)\left(E_{2}+E_{3}\right)-2 i F_{1}(\overline{a b})\right)=\phi(A)\left(E_{2}+E_{3}\right) \\
& =\phi(A) \beta\left(E_{2}+E_{3}\right) .
\end{aligned}
$$

Thus (i) is true for $X=E_{2}, E_{3}$. For $X=E_{1}$, (i) is trivial.

$$
\begin{aligned}
\beta \phi(A) F_{1}(z) & =\beta \phi(A) F_{1}(s+t) \quad\left(z=s+t \in C^{C} \oplus\left(C^{\perp}\right)^{C}=\left(^{C}\right)\right. \\
& =\beta\left(-2 i(s, \overline{b a})\left(E_{2}+E_{3}\right)+F_{1}\left(a^{2} s-\bar{b}^{2} \bar{s}\right)+F_{1}(t)\right) \\
& =-2 i(s, \overline{b a})\left(E_{2}+E_{3}\right)+F_{1}\left(a^{2} s-\bar{b}^{2} \bar{s}\right)+\left(\xi\left(E_{2}-E_{3}\right)+F_{1}\left(t^{\prime}\right)\right)
\end{aligned}
$$

(for some $\xi \in C, t^{\prime} \in\left(C^{\perp}\right)^{C}$ )

$$
=\phi(A)\left(F_{1}(s)+\xi\left(E_{2}-E_{3}\right)+F_{1}\left(t^{\prime}\right)\right)=\phi(A) \beta F_{1}(s+t)=\phi(A) \beta F_{1}(z) .
$$

$$
\begin{aligned}
\beta \phi(A) F_{2}(z) & =\beta\left(F_{2}(\bar{a} z)-i F_{3}(\bar{z} b)\right) \\
& =4 \beta\left(\left(F_{1}(1) \times F_{2}(z)\right) \times F_{1}(a)\right)-4 i \beta\left(\left(F_{1}(1) \times F_{3}(\bar{z})\right) \times F_{1}(\bar{b})\right) \\
& =4\left(\beta F_{1}(1) \times \beta F_{2}(z)\right) \times \beta F_{1}(a)-4 i\left(\beta F_{1}(1) \times \beta F_{3}(\bar{z})\right) \times \beta F_{1}(\bar{b})
\end{aligned}
$$

(put $\beta F_{2}(z)=F_{2}(x)+F_{3}(y), x, y \in \mathbb{C}^{c}$, then $\beta F_{3}(\bar{z})=\beta\left(2 F_{1}(1) \times F_{2}(z)\right)=2 F_{1}(1) \times \beta F_{2}(z)$ $\left.=2 F_{1}(1) \times\left(F_{2}(x)+F_{3}(y)\right)=F_{2}(\bar{x})+F_{3}(\bar{y})\right)$

$$
\begin{aligned}
& =4\left(F_{1}(1) \times\left(F_{2}(x)+F_{3}(y)\right)\right) \times F_{1}(a)-4 i\left(F_{1}(1) \times\left(F_{3}(\bar{x})+F_{2}(\bar{y})\right)\right) \times F_{1}(\bar{b}) \\
& =F_{2}(\bar{a} x)+F_{3}(y \bar{a})-i\left(F_{3}(\bar{x} b)+F_{2}(b \bar{y})\right) \\
& =F_{2}(\bar{a} x)-i F_{3}(\bar{x} b)-i F_{2}(b \bar{y})+F_{3}(y \bar{a}) \\
& =\phi(A) F_{2}(x)+\phi(A) F_{3}(y)=\phi(A)\left(F_{2}(x)+F_{3}(y)\right)=\phi(A) \beta F_{2}(z) .
\end{aligned}
$$

Similarly, $\beta \phi(A) F_{3}(z)=\phi(A) \beta F_{3}(z)$. Thus (i) is proved.

LEMMA 5.4. $\phi(S U(2))$ and $\operatorname{Spin}(7)$ are contained in $\left(E_{6}\right)^{\tau \sigma_{3}}$.

ProOF. $\phi(S U(2)) \subset\left(E_{6}\right)^{\tau \sigma_{3}}$ is clear, noting that $\left(\begin{array}{rr}-e & 0 \\ 0 & e\end{array}\right)\left(\begin{array}{rr}a & -\bar{b} \\ b & \bar{a}\end{array}\right)\left(\begin{array}{rr}-e & 0 \\ 0 & e\end{array}\right)^{-1}$ $=\left(\begin{array}{rr}a & \bar{b} \\ -b & \bar{a}\end{array}\right), \tau i=-i$ and $b, i$ appear simultaniously in $\phi(A) X . \quad$ Next, $\beta \in \operatorname{Spin}(7)$ $\subset F_{4}$ implies $\tau \beta=\beta \tau$ and $\sigma_{3} \beta=\beta \sigma_{3}$ (Lemma 5.3). Hence $\operatorname{Spin}(7) \subset\left(E_{6}\right)^{\tau \sigma_{3}}$.

THEOREM 5.5. $\left(E_{6}\right)^{\tau \sigma_{3}} \cong(S U(2) \times \operatorname{Spin}(7)) / \boldsymbol{Z}_{2}, \boldsymbol{Z}_{2}=\{(E, 1),(-E, \boldsymbol{\sigma})\}$.

ProOF. We define a mapping $\phi: S U(2) \times \operatorname{Spin}(7) \rightarrow\left(E_{6}\right)^{\tau \sigma_{3}}$ by

$$
\phi(A, \beta)=\phi(A) \beta \text {. }
$$

Then $\psi$ is well-defined (Lemma 5.4) and is a homomorphism (Lemma 5.3). We 
shall show that $\phi$ is onto. (Although it suffices to show $\operatorname{dim}\left(\left(e_{6}\right)^{\tau \sigma_{3}}\right)=24$, we will give a direct proof). Let $\alpha \in\left(E_{6}\right)^{\tau \sigma_{3}}$. Then we can put

$$
\alpha\left(i\left(E_{2}+E_{3}\right)\right)=i \eta\left(E_{2}+E_{3}\right)+F_{1}(s), \quad \eta^{2}+|s|^{2}=1, \eta \in \boldsymbol{R}, s \in \boldsymbol{C} .
$$

In fact, since $\alpha \in\left(E_{6}\right)^{\tau \sigma_{3}} \subset\left(E_{6}\right)_{E_{1}}=\operatorname{Spin}(10)$ (Lemma 5.2), we can put $\alpha\left(i\left(E_{2}+E_{3}\right)\right.$ ) $=\xi E_{2}-\tau \xi E_{3}+F_{1}(s+t), \xi \in C, s+t \in \boldsymbol{C} \oplus \boldsymbol{C}^{\perp}=\boldsymbol{C}$. From $\tau \sigma_{3} \alpha=\alpha \tau \sigma_{3}$, we have $\xi \in i \boldsymbol{R}, t=0$. And $2\left(\eta^{2}+|s|^{2}\right)=\left\langle\alpha\left(i\left(E_{2}+E_{3}\right)\right), \alpha\left(i\left(E_{2}+E_{3}\right)\right)\right\rangle=\left\langle i\left(E_{2}+E_{3}\right), i\left(E_{2}+E_{3}\right)\right\rangle$ $=2$. Now put

$$
P=\frac{1}{\sqrt{2(1-\eta)}}\left(\begin{array}{cc}
\bar{s} & 1-\eta \\
-1+\eta & s
\end{array}\right) \quad(\text { if } \eta=1, \text { put } P=E) .
$$

Then $P \in S U(2)$ and $\phi(P)\left(i \eta\left(E_{2}+E_{3}\right)+F_{1}(s)\right)=i\left(E_{2}+E_{3}\right)$. Hence $\phi(P) \alpha\left(i\left(E_{2}+E_{3}\right)\right)$ $=i\left(E_{2}+E_{3}\right)$, that is, $\delta=\phi(P) \alpha \in\left(F_{4}\right)_{E_{1}}=\operatorname{Spin}(9)$, moreover $\left((\operatorname{Spin}(9))^{\tau \sigma_{3}}=(\operatorname{Spin}(9))^{\sigma_{3}} \subset\right.$ $(\operatorname{Spin}(10))^{\sigma_{3}}$. Hence we can put

$$
\delta F_{1}(1)=F_{1}\left(s_{0}\right), \quad \delta F_{1}(e)=F_{1}\left(e s_{0}\right), \quad s_{0} \in C,\left|s_{0}\right|=1
$$

(Lemma 3.4). Choose $a \in C$ such that $a^{2}=\bar{s}_{0}$ and put $\beta=D_{a} \delta$, then $\beta\left(i\left(E_{2}+E_{3}\right)\right.$ ) $=i\left(E_{2}+E_{3}\right), \beta F_{1}(1)=F_{1}(1), \beta F_{1}(e)=F_{1}(e)$, that is, $\beta=D_{a} \phi(P) \alpha \in \operatorname{Spin}(7)$. Therefore we have a presentation such that

$$
\alpha=\phi\left(P^{-1} A\right) \beta, \quad P^{-1} A \in S U(2) \text { (where } A=\left(\begin{array}{ll}
\bar{a} & 0 \\
0 & a
\end{array}\right), \beta \in \operatorname{Spin}(7) .
$$

Thus $\phi$ is onto. $\operatorname{Ker} \phi=Z_{2}=\{(E, 1),(-E, \sigma)\}$. In fact, let $\phi(A) \beta=1, A=$ $\left(\begin{array}{rr}a & -\bar{b} \\ b & \bar{a}\end{array}\right) \in S U(2), \beta \in \operatorname{Spin}(7)$. Then $E=\phi(A) \beta E=\phi(A) E=E_{1}+\left(|a|^{2}-|b|^{2}\right)\left(E_{2}+E_{3}\right)$. Hence $|a|^{2}-|b|^{2}=1$. Together with $|a|^{2}+|b|^{2}=1$, we have $b=0$, so $A=\left(\begin{array}{ll}a & 0 \\ 0 & \bar{a}\end{array}\right)$. From $F_{1}(1)=\phi(A) \beta F_{1}(1)=\phi(A) F_{1}(1)=F_{1}\left(a^{2}\right)$, we have $a^{2}=1$, hence $a= \pm 1$, so $A= \pm E$. Then $\beta=\phi(E)=1$ or $\beta=\phi(-E)=\sigma$. Thus we have the required isomorphism.

\section{References}

[1] Helgason, S., Differential geometry, Lie groups and symmetric spaces, Akademic Press, New York, 1978.

[2] Yokota, I., Simply connected compact simple Lie group $E_{6(-78)}$ of type $E_{6}$ and its involutive automorphisms, J. Math. Kyoto Univ., 20 (1980), 447-473.

[3] Yokota, I., Realizations of automorphisms $\sigma$ of order 3 and $G^{\sigma}$ of compact exceptional Lie groups $G, \mathrm{I}, G=G_{2}, F_{4}, E_{6}$, J. Fac. Sci. Shinshu Univ., 20 (1985), 131-144.

[4] Yokota, I., Realizations of involutive automorphisms $\sigma$ and $G^{\sigma}$ of exceptional linear 
Lie groups $G$, part I, $G=G_{2}, F_{4}$ and $E_{6}$, Tsukuba J. Math., 14 (1990), 185-223. [5] Yokota, I., Ishihara, T. and Yasukura, O., Subgroup $\left((S U(3) \times S U(6)) / \boldsymbol{Z}_{2}\right) \cdot \boldsymbol{Z}_{3}$ of the simply connected compact simple Lie group $E_{7}$, J. Math. Kyoto Univ., 23 (1983), 715-747.

Ichiro Yokota

Department of Mathematics

Shinshu University

Asahi, Matsumoto

Nagano, Japan
Osamu Shukuzawa

Department of Mathematics

Yamanashi University

Takeda, Kofu

Yamanashi, Japan 